\title{
Role of caffeic and chlorogenic acid in the modulation of cellular fatty acid uptake
}

\author{
Mirko Marino ${ }^{1}$, Massimiliano Tucci ${ }^{1}$, Valentina Taverniti ${ }^{1}$, Patrizia Riso ${ }^{1}$, Marisa Porrini ${ }^{1}$, \\ Dorothy Klimis-Zacas ${ }^{2}$ and Cristian Del Bo'1 \\ ${ }^{1}$ Department of Food, Environmental and Nutritional Sciences, Università degli Studi di Milano, Milano, Italy and \\ ${ }^{2}$ School of Food and Agriculture, University of Maine, Orono, USA
}

\begin{abstract}
Polyphenols are bioactive molecules widely distributed in numerous foods such as fruits, vegetables, tea, coffee, cocoa and beverages. Their main classification include flavonoids (i.e. flavonols, flavones, flavanones, flavanols, anthocyanins, and isoflavones), non-fl avonoids (i.e. lignans and stilbens) and phenolic acids (i.e. hydroxycinnamic and hydroxybenzoic acids) ${ }^{(1)}$. Caffeic acid (CA) and chlorogenic acid (CGA; an ester of CA and quinic acid) are the major representatives of hydroxycinnamic acids. Accumulating evidence has demonstrated that CA and CGA may exert different biological activities, including antioxidant, anti-inflammatory, antidiabetic, and antihypertensive ${ }^{(2)}$. Despite these promising and diverse anti-atherosclerotic actions, investigations addressing the effect of CA and CGA on atherogenesis are scarce.

The present study evaluated the capacity of CA and CGA to reduce lipid accumulation in macrophages derived from monocytic THP-1 cells. THP-1-derived macrophages were incubated with fatty acids (500 $\mu \mathrm{M}$ oleic/palmitic acid, $2: 1 \mathrm{ratio})$ and different concentrations (from 0.03 to $3 \mu \mathrm{M}$ ) of CA and CGA, alone or in combination. Lipid accumulation was quantified spectrophotometrically (excitation: $544 \mathrm{~nm}$, emission: $590 \mathrm{~nm}$ ) with the fluorescent dye, Nile red. The fold increase compared to the control (without fatty acids) was calculated. In addition, the expression of several transcription factors including peroxisome proliferator-activated receptor gamma (PPAR $\gamma)$ and CCAAT/enhancer-binding protein (CEBP), as potential mechanisms involved in the regulation of lipid accumulation, was evaluated by real time PCR.

Analysis of variance (ANOVA) was used to assess the effect of the different concentrations of CA and CGA on lipid accumulation in THP-1 macrophages following stimulation with FA.

The preliminary results obtained have shown a significant increase in lipid accumulation following fatty acid exposure $(\mathrm{p}<0.0001)$. Incubation with CA and CGA did not reduce lipid accumulation in THP-1 derived macrophages, while the combination of CA + CGA at $0.03,0.3$ and $3 \mu \mathrm{M}(\mathrm{p}<0.01)$ decreased cellular fatty acid uptake at all concentrations tested by $-28 \%,-32 \%,-23 \%$, respectively. An apparent modulation of the transcriptional activity of PPAR $\gamma$, but not CEBP, was observed following the combination of phenolic acids.

In conclusion, the incubation of CA + CGA at physiologically relevant concentrations, but not the single compounds, seem to reduce the uptake of fatty acids in THP-1-derived macrophages. Further experiments are ongoing in order to confirm the findings obtained and to better identify the mechanisms of action involved in the reduction of lipid accumulation as a key phenomenon of atherogenesis.
\end{abstract}

\section{Conflict of Interest}

There is no conflict of interest.

\section{References}

1. Antioxid Redox Signal 2013, 18, 1818-1892.

2. Eur J Nutr. 2017, 56, 2215-2244. 